\title{
Facebook comme plate-forme de développement des compétences professionnelles des enseignants et des étudiants de langue: une étude de cas
}

\author{
Facebook as a platform for developing professional \\ skills of language teachers and students: case study
}

\author{
María Eleni Katsinopoulou \\ Université de Chypre \\ mkatsinopoulou@hotmail.com
}

\begin{abstract}
Nowadays, the diffusion of technologies, and in particular social networking, requires language teachers to learn new data in their teaching practices. Yet, by observing on the net, we remark the existence of many communities, especially on Facebook, which aim to inform teachers and language students about new knowledge. What are the reasons why a language teacher or student becomes a member of these communities? Do social networking sites help language teachers improving their professional skills? Developing professional skills is one of the most significant actions required for a language student to secure a teaching career.
\end{abstract}

\section{Key-words}

Facebook, language teachers, social networking, professional skills.

\begin{abstract}
Resumen
En la actualidad, la difusión de tecnologías $\mathrm{y}$, en particular, la creación de redes sociales, exige que los profesores de idiomas aprendan nuevos datos en sus prácticas docentes. Sin embargo, al observar la red, comentamos la existencia de muchas comunidades, especialmente en Facebook, cuyo objetivo es informar a los profesores y a los estudiantes de idiomas acerca de los nuevos conocimientos. ¿Cuáles son las razones por las que un profesor de idiomas o un estudiante se convierte en miembro de estas comunidades? ¿Los sitios de redes sociales ayudan a los profesores de idiomas a mejorar sus aptitudes profesionales? El desarrollo de habilidades profesionales es una de las acciones más importantes que se necesitan para que un estudiante de idiomas obtenga una carrera docente.
\end{abstract}

\section{Palabras clave}

Facebook, profesores de idiomas, redes sociales, aptitudes profesionales. 


\section{Introduction}

De nos jours, la propagation des technologies et plus particulièrement, du réseautage social imposent aux enseignants de langues de nouvelles données dans leurs pratiques d'enseignement. En naviguant sur le net, nous constatons l'existence de beaucoup de communautés, notamment sur Facebook, qui ont pour but d'informer les enseignants et les étudiants de langue sur de nouvelles connaissances. Par la suite, nous étudierons si les enseignants et les futurs enseignants rejoignent des groupes sur Facebook, qui concernent leur Université, même après avoir obtenu leur diplôme. Quelles sont, alors, les raisons pour lesquelles un enseignant ou un étudiant de langues devient membre de ces communautés? Le réseautage social aide-t-il les enseignants de langue à améliorer leurs compétences professionnelles?

La formation personnelle des enseignants de langue joue un rôle important pour améliorer les connaissances et les compétences d'un professeur comme le soulignent Sylla et De Vos (2010): "Le développement professionnel met l'accent sur l'engagement de l'individu à parfaire ses connaissances et ses compétences, tout au long de sa carrière". Les discussions à distance qui se réalisent parmi les membres des groupes sur Facebook, le réseau social dont nous allons nous occuper dans cet article, nous permettent d'observer des débats virtuels dont la solution contribue au progrès professionnel des enseignants (Daele, 2010).

\section{Communautés de pratique et réseautage social}

D'après Dillenbourg et al., (2003), la communauté est un groupe de personnes qui ont les mêmes intérêts et qui se ressemblent pour acquérir des connaissances. Les communautés numériques sont réelles, c'est-à-dire, elles comprennent de vraies personnes et le mode de communication utilisé repose sur les outils de communication électronique. Selon Brown et al., (1989), traduction de Sarré (2012), la notion de communauté de pratique se réfère au concept de l'apprentissage. Cependant, l'apprentissage existe sous certaines formes de coparticipation et l'apprenant peut acquérir des connaissances et développer ses compétences professionnelles et éducatives. Lave \& Wenger (1991) ont développé le principe de "participation périphérique légitime" qui est un mode d'intégration d'un novice (apprenant) à une communauté de pratique. Tout d'abord, l'apprenant "entre" dans une communauté comme observateur et par la suite il s'intègre, au fil de l'évolution de son apprentissage, dans la culture du groupe.

D’après Wenger (2005), les communautés en ligne consistent à des regroupements facilitant la réalisation de projets et une forme de participation pouvant être identifiée à une compétence. Le but des communautés de pratique est la capacité individuelle et collective d'être en contact avec le monde réel. Selon Wenger: 
La théorie des communautés de pratique propose de concevoir l'apprentissage sous l'angle d'une participation sociale. En ce sens précis, la participation ne se limite pas à un engagement dans certaines activités courantes avec d'autres personnes, elle réfère également au processus plus englobant de 'collaboration active aux pratiques d'une communauté sociale' et de la construction d'identités en lien avec elle. Ainsi, participer à une équipe de travail ou à une clique constitue à la fois une forme d'appartenance à une forme d'activité. (Wenger, 2005, traduction de Fernand Gervais, 2005: 2)

L'objectif des membres d'une communauté de pratique est de "développer et d'enrichir la pratique professionnelle grâce au partage et à la mise en commun des connaissances complémentaires de ses membres" (Henri \& Pudelko, 2006: 118). Depover \& Marchand, (2002) remarquent que l'activité du groupe est une succession d'actions produites par plusieurs membres de la communauté. Il pose une question, il élabore un texte et il participe aux discussions. De cette manière, les membres d'un groupe peuvent progresser plus facilement dans la cognition individuelle dans son ensemble. Un individu, en consultant une communauté de pratique, peut acquérir des connaissances organisées et procéder à l'action collective, ce qui est très important, notamment dans sa formation professionnelle. Cobb (1994) et Cobb et Yackel (1996) prétendent que l'individu est responsable de l'apprentissage et que la communauté de pratique est le "moyen" par lequel les individus peuvent y accéder. Les communautés offrent un "environnement" sécurisé pour les individus, où ils peuvent apprendre à l'aide de l'observation, de l'interaction et de la discussion avec les collègues les plus expérimentés.

\section{Pourquoi s'inscrire à une communauté en ligne?}

Un individu s'inscrit à une communauté de pratique sur le net, il est représenté par un artefact (une adresse électronique, un avatar, etc.) et de cette manière il peut participer aux échanges. Les communautés de pratique donc, encouragent l'échange des opinions et des idées. De plus, dans une communauté de pratique la participation des individus est associée à la réalisation des interactions. Les membres d'une communauté de pratique partagent une réciprocité des influences qui se crée dans la communauté. Dès que les membres fréquentent la communauté de pratique, des interactions se développent entre eux. Il s'agit d'un “espace social" des relations sociales qui sont ancrées dans les valeurs, les règles, les rôles, les croyances et les idéaux de cette communauté. Selon Yorks (2005), l'espace social est un lieu où nous pouvons échanger, créer et utiliser des connaissances. Dans le modèle de Kreijns et al. (2002) et Kreijns et al., (2007); dans Daele (2010): 69, la sociabilité d'un environnement collectif virtuel donne la possibilité aux participants de soutenir les interactions sociales, mais aussi il rend les relations interpersonnelles plus faciles et crée, de même, la confiance, la cohésion sociale ou le sentiment d'appartenance au groupe sur le net. Selon Daele (2010), 
les trois variables (sociabilité, techniques pédagogiques et présence sociale) conditionnent l'émergence et la durabilité des interactions sociales en ligne.

D'après Sylla et De Vos (2010), la participation au groupe peut être active pour certains et passive pour autres. Cela montre l'implication des membres à la communauté. Plus précisément, les membres actifs posent des questions et partagent leurs expériences avec leurs collègues. De cette façon, les pratiques utilisées sont mises à disposition pour tout à chacun et peuvent être adaptées ou négociées. Les membres qui sont moins actifs ou qui constituent comme Wenger le nomme "la couche périphérique" peuvent lire ces expériences et les débats virtuelles et changer ou améliorer leur pratique. Cette participation ne peut pas être caractérisée passive, même si elle n'offre pas d'expressions publiques ou explicites (Wenger et al., 2002). Quand les membres moins actifs ont le besoin d'interagir, ils préfèrent utiliser les messageries privées. Ils peuvent échanger, alors, des points de vue avec certains de leurs collègues. Nous constatons, donc, que la collaboration, la réflexion et l'interaction offertes par la communauté de pratique, influencent même les personnes moins actives.

\section{Facebook et son rôle au développement des compétences professionnelles}

Facebook est un site, qui joue un rôle significatif à la communication et à l'acquisition des connaissances. Précisément, en Grèce, qui est le pays de référence de notre étude, Facebook est utilisé largement au domaine de l'éducation et c'est le réseautage social qui a le plus grand nombre de participants. Mousa (2018: 67) écrit que:

Facebook est un site social où l'on peut poster des évènements de sa vie personnelle et à travers lequel on pourrait partager des quantités innombrables d'informations [...] Donc, FB pourrait être considéré comme un outil d'apprentissage puissant qui a permis une extension des outils communicatifs vers des horizons non expérimentés auparavant.

Selon Gunawardena et al., (2009) les médias sociaux développent l'interaction, la collaboration et la contribution. Une caractéristique essentielle des médias sociaux est que les utilisateurs peuvent échanger des documents et créer ou construire des connaissances qui sont dépendantes de l'intelligence collective des utilisateurs. Les médias sociaux comme Facebook, améliorent l'interaction sociale et les relations parmi les utilisateurs, pouvant ainsi y participer régulièrement et créer donc " une vie " sur l'écran (Fine, 1987). Selon Blattner et Lomicka (2012: 4),

Facebook offers a wide array of technological affordances supporting a diverse range of interests and practices which integrate several modes of Computer Mediated Communication (CMC) such as self-presentation, and one-to-one or one-to-many written exchanges. 
Blattner et Lomicka (2012) continuent en écrivant qu'à l'aide du réseautage social les membres ont la possibilité de décrire et d'échanger de plusieurs informations concernant leur vie. De cette façon, les auteurs soutiennent que les enseignants ont commencé à considérer Facebook comme un outil utile à la pédagogie des langues, mais aussi dans l'éducation supérieure. Selon Godwin-Jones, Facebook "has the potential to find means to link informal and recreational writing with academic writing” (Godwin-Jones, 2008: 7). D'après Daele (2014: 1)

Avec l'avènement de l'Internet grand public dans les années 1990, des initiatives variées ont vu le jour pour proposer aux enseignants des formations continues recourant à des interactions à distance entre collègues (Dede, 2006) ou des modes de communication plus informels comme les communautés virtuelles. (Daele \& Charlier, 2006)

D'autre part, le développement professionnel d'un enseignant, selon Sylla et De Vos (2010) comporte deux approches qui nous intéressent. Premièrement, l'approche du "développement personnel" (personal development), selon laquelle le développement professionnel de l'enseignant est considéré comme le résultat d'un processus de développement personnel. Les caractéristiques de la personnalité de l'enseignant, naturellement, jouent un rôle important, comme l'autonomie, la tolérance, la flexibilité, la compréhension des différences individuelles entre les élèves. Ensuite, l'approche de la "professionnalisation" de l'enseignant (teacher professional approach): le développement professionnel de l'enseignant est le résultat de l'acquisition des savoirs et des compétences. Dans cette approche la base de la formation professionnelle est l'interaction avec l'environnement. La participation d'un enseignant à une communauté est liée au développement personnel, à l'adaptation de l'enseignant dans le milieu scolaire et à l'acquisition des savoirs et compétences. Certains auteurs (Butler, 2005; Hoekstra et al., 2009) constatent que le développement professionnel comporte une dimension sociale par la confrontation des pratiques, mais aussi, une dimension individuelle qui est généralement appelée “apprentissage auto-régulé”. C'est-à-dire qu'une personne peut auto-réguler son apprentissage dans la mesure où elle prend part activement dans les situations d'apprentissage dans lesquelles elle participe, et ceci d'un point de vue cognitif, métacognitif ou motivationnel (Greene \& Azevedo, 2007). L'entrée et la participation d'un enseignant à une communauté de pratique est une démarche active afin de chercher des informations nouvelles dans le domaine de l'enseignement ou discuter de sujets pédagogiques comme par exemple: des méthodes, des représentations ou des pratiques de classe. D'après Charlier (2006), le réseau professionnel est devenu une nécessité dans la carrière d'un enseignant de l'enseignement supérieur dans sa formation professionnelle et dans son travail en équipe. A cet égard, Daele et Charlier (2006) déduisent qu'il y a cinq processus, comme les échanges, le partage d'expériences, l'analyse, le débat et la création de nouvelles méthodes qui peuvent conduire l'utilisateur-enseignant à la formation professionnelle. Il faut, ainsi, ajouter l'importance de l'entrée des enseignants dans la communauté, leur participa- 
tion et le niveau de leurs savoirs. Finalement, il faut observer l'importance du concept de la collaboration. D'après Charlier (2006), la collaboration peut se réaliser au sein d'une communauté de pratique. De cette façon, les participants peuvent développer et mettre en œuvre un dispositif de formation en commun.

Le développement professionnel des enseignants est formé de plusieurs sujets différents, comme la réflexion et l'action (Schön, 1983, 1987) ou l'interaction avec ses pairs, dans diverses situations, professionnelles ou privées (Day, 1999) dans les espaces numériques (Depover \& Marchand, 2002) au sein de communautés virtuelles (Audran \& Simonian, 2003; Daele, 2004). De plus, la formation professionnelle des enseignants est basée sur l'apprentissage collaboratif (Charlier et Peraya, 2003). Donnay et Charlier (2006) continuent en écrivant que le développement professionnel des enseignants doit être dynamique et continu, c'est-à-dire que, le développement professionnel est un processus continuel et que les apprentissages acquis sont réinvestis dans les situations professionnelles quotidiennes. Beaucoup de chercheurs et formateurs (Collinson et al., 2009; Hou, Sung, \& Chang, 2009; $\mathrm{Ng} \&$ Tan, 2009) affirment l'importance des échanges des idées et des débats pour le développement professionnel et l'amélioration des pratiques d'enseignement. D'après Daele (2014), la participation d'enseignants à des débats virtuels peut conduire au développement professionnel en faisant l'association de débats virtuels, de conditions individuelles et d'interaction qui mènent à une réflexion sur leurs pratiques d'enseignement. Selon Charlier (1998) et Huberman, (1986) le but de la formation professionnelle inclut l'accès aux savoirs et compétences professionnelles d'autres enseignants et la validation de leurs propres pratiques.

\section{Interactions sur le réseautage social}

Ellison et al., (2009) observent que les sites de réseautage social ont la possibilité de changer nos vies sociales. La plupart des utilisateurs sont des jeunes et nous pouvons observer les échanges à l'interaction et aux connections sociales. Blattner et Lomicka (2012) affirment que le réseautage social offre aux étudiants de langues l'occasion de communiquer plus facilement avec d'autres étudiants de la même année qu'eux ou même avec leurs enseignants. De nos jours, cette communication est devenue plus personnelle et peut être un motif dans le progrès de leurs études. Les écrivains Mazer et al., (2007) affirment que ce type d'interaction peut s'avérer positif dans les relations étudiant-étudiant, mais aussi étudiant-enseignant. Cette connexion peut, éventuellement, mener à un apprentissage profond. Les apprenants acquièrent ainsi, une plus grande autonomie et peuvent participer activement au processus de l'apprentissage à l'aide de l'utilisation du réseautage social puisqu'ils ont le contrôle de tout ce qu'ils veulent apprendre. Selon Karsenti et al., (2007); Deaudelin et al., (2003), la collaboration est une source d'aide au processus d'autoformation qui exerce l'autonomie de l'enseignant et de l'étudiant et construit des connaissances de façon collective. Le réseautage 
social, et plus précisément Facebook, peut devenir une source d'éducation, mais parfois les apprenants ne connaissent pas exactement son utilité en ce qui concerne le domaine de l'éducation. C'est pour cette raison qu'ils ont besoin d'aide afin de comprendre la grande gamme des possibilités offertes par Facebook.

À ce point précis, nous devons également, indiquer l'importance de débats virtuels et de controverse. Daele (2013) a décrit les quatre catégories des conditions d'interactions sociales à distance impliquant un débat:

1. Conditions et caractéristiques individuelles de départ: Les attitudes et les compétences sociales et cognitives des participants dans des débats sont inextricablement liées aux compétences d'utilisation des technologies du groupe. Les participants doivent connaitre la façon d'argumenter dans un débat et la manière de s'exprimer dans les interactions. Ce sont des attitudes et compétences très importantes pour les participants, afin qu'ils puissent participer aux débats et se sentir à l'aise avec les autres collègues. Ensuite, l'étape de carrière est un élément très signifiant qui aide l'assurance personnelle envers ses propres pratiques et conceptions. Les enseignants - novices n'ont pas la même expérience d'enseignement que les collègues les plus expérimentés. De cette manière, ils sont plus hésitants à participer aux débats pédagogiques. De plus, il y a des compétences importantes pour les enseignants du groupe afin de prendre part aux débats virtuels. Ces compétences se réfèrent à la conscience de ses propres compétences d'enseignement, à la connaissance des moyens pour leur développement personnel et à la reconnaissance des instants et des moyens les plus propices pour améliorer l'apprentissage de leur métier.

2. Conditions "caractéristiques et représentations individuelles des discussions en ligne": Les caractéristiques des interactions à distance: l'empathie exprimée, l'intensité de la relation, l'approfondissement des arguments, le sujet de discussion en lien avec les intérêts des participants, etc. Les compétences communicationnelles de chaque participant jouent un rôle important et peuvent influencer ces caractéristiques. De plus, l'auteur insiste sur la méta-communication exprimée au sein de la discussion (humour, émoticons, explicitation de la façon de lire ou comprendre les messages, etc.). Il y a des personnes qui ne connaissent pas l'utilisation des émoticons ou des codes et pour cette raison les participants doivent s'exprimer de façon explicite. Les participants aux discussions doivent avoir une présence sociale exprimée et ressentie par les autres collègues. Tous les collègues, alors, auront un sentiment de "confiance" et la possibilité de s'exprimer librement.

3. Conditions "perception de soi au sein de la communauté": Les participants doivent expliquer clairement leur projet personnel, c'est-à-dire, les raisons pour lesquelles ils participent au groupe. Ils peuvent, alors, repérer les éléments qui les intéressent. Les enseignants doivent avoir le sentiment de sécurité, c'est-à-dire, la possibilité de s'exprimer librement et de commettre des erreurs sans être critiqués par d'autres collègues. C'est un élément très significatif pour les enseignants-novices qui ne sont pas sûrs de leurs pratiques. Ensuite, un 
autre élément important est le sentiment d'appartenance à la communauté. Il faut que les participants soient intégrés à la communauté et de cette manière, y apporter leurs connaissances et leurs compétences. La reconnaissance et la valorisation dans les débats sont très importantes pour le développement du sentiment d'appartenance. De plus, les enseignants doivent avoir une curiosité personnelle pour les sujets de discussion de la communauté. La curiosité aide les membres du groupe à participer aux discussions. La communauté doit constituer un lieu d'apprentissage, un lieu où les interactions et leurs produits acquièrent de la valeur. Il faut que les participants aient le sentiment que les débats, auxquels ils participent, puissent les aider dans leur formation professionnelle. Finalement, la composition de la communauté doit aider les membres à interagir avec les autres, à partager les mêmes préoccupations pour que les participants puissent s'investir et remettre en cause leurs pratiques ou leurs conceptions. Ils peuvent, alors, les changer si cela est nécessaire.

4. Condition "soutien à la communauté": Le rôle de l'animateur-modérateur est significatif dans la communauté parce qu'il peut lancer et relancer des sujets de discussion. Cette personne peut assurer l'existence et le respect des règles d'interaction. L'animateur peut être considéré comme une source pour la communauté et les membres participeront plus volontiers à distance.

Finalement, il ne faut pas oublier que les utilisateurs de Facebook via une communauté de pratique peuvent améliorer leurs compétences communicatives dans la langue seconde et se connecter avec des personnes natives de cette langue (Blattner et Fiori, 2009). De plus, selon Karpati (2009), le réseautage social facilite les enseignants à promouvoir l'apprentissage collaboratif et met leurs apprenants au centre de leur intérêt. Mills (2011) écrit que les apprenants utilisent Facebook comme un complément à leur environnement scolaire. Les outils interactifs, qu'ils utilisent, les aident à échanger une réflexion collective et ils peuvent accéder à des sources qui améliorent les sujets discutés en classe.

\section{Une étude de cas}

Afin que nous constations les fondements théoriques et leur pratique actuelle nous avons distribué un questionnaire ( $c f$. Annexe) auprès des enseignants et des étudiants de langues étrangères et plus précisément de la langue française et espagnole à certains groupes sur Facebook. Afin d'avoir un échantillon représentatif, nous avons publié le questionnaire plusieurs fois à chaque groupe sur Facebook. Plus précisément, les communautés de pratique que nous avons utilisées sont: "Professeurs de la langue française", "Professeurs de la langue espagnole", "Langue/Littérature Françaises Université Aristote”, "Langue/Littérature Espagnole”. Nous avons choisi ces groupes grâce au nombre des participants. Plus spécialement, chaque groupe comprend: 
- $\quad 1.425$ Personnes appartenant au groupe: "Professeurs de la langue française" (https://www.facebook.com/groups/143927088986355/ [05/9/2020].

- 810 Personnes appartenant au groupe: "Professeurs de la langue espagnole" (https://www.facebook.com/groups/122766374441921/ [05/9/2020].

- $\quad 1.540$ Personnes appartenant au groupe: "Langue/Littérature Françaises Université Aristote” (https:/www.facebook.com/groups/220256644694442/ [05/9/2020].

- 479 Personnes appartenant au groupe: "Langue/Littérature Espagnole” https:// www.facebook.com/groups/172974942737025/ [05/9/2020].

Finalement, nous avons reçu 68 réponses. En général, le questionnaire était bien structuré et notre enquête a été réalisée de juillet 2020 jusqu'à septembre 2020.

Plus concrètement, notre questionnaire ( $c f$. Annexe) contient:

- des questions fermées (oui/non) où la personne interrogée doit choisir la réponse qui lui convient (questions 10, 12, 17 et 19).

- des questions à choix multiple où l'interrogé peut choisir plus d'une réponse parmi les options offertes (questions 2, 3, 4, 5, 7 et 15).

- une question avec l'utilisation de l'échelle Likert où les enseignants/étudiants doivent exprimer le degré d'utilité des quelques propositions de 1 (pas utile) 3 (très utile).

- des questions ouvertes où la personne interrogée peut s'exprimer librement sans aucune guidance (questions 1, 6, 8, 9, 11, 13, 16, 18, 20 et 21).

À l'aide du cas d'étude nous avons eu la possibilité d'analyser les réponses des participants et à s'en délecter. Par la suite, l'analyse du discours nous a permis d'étudier les réponses des participants mot à mot et de tirer nos conclusions. Cette approche méthodologique nous donne la possibilité d'analyser le contexte et le contenu du discours oral ou écrit.

Plus concrètement, Maingueneau (1998: 2) définit l'analyse du discours:

L'analyse de discours est l'analyse de l'articulation du texte et du lieu social dans lequel il est produit. Le texte seul relève de la linguistique textuelle; le lieu social, lui, de disciplines comme la sociologie ou l'ethnologie. Mais l'analyse de discours en étudiant le mode d'énonciation, se situe elle à leur charnière.

Encore, nous avons utilisé le cas d'étude qui fait partie de la méthodologie qualitative et quantitative, aussi. Selon Orum et al., "A case study is here defined as an in-depth, multifaceted investigation, using qualitative research methods, of a single social phenomenon. The study is conducted in great detail and often relies on the use of several data sources" (Orum et al., 1991, 2). Loiselle \& Harvey (2007) soutiennent que "les recherches appliquées, orientées 
vers l'action, ne constituent pas une simple application des connaissances fondamentales à un contexte particulier" (Loiselle et Harvey, 2007, 50) et ajoutent que la démarche inductive est plus appropriée pour l'analyse du discours parce ce qu'avec cette approche, nous pouvons comprendre mieux les données recueillies. En effet, suivant une approche inductive, "le chercheur-développeur, dans son processus de décision visant à raffiner le produit, considéra dans ses choix de développement ses propres réflexions, les perceptions, réflexions et actions des participants, et les éléments tirés de l'analyse des écrits" (ibid., 50).

\section{Analyse des données recueillis}

En répondant à notre question, si la fréquentation aux communautés de pratique sur Facebook améliore les compétences professionnelles des enseignants et des étudiants de langues étrangères, nous avons constaté qu' en réalité la fréquentation aux groupes est une condition préalable afin que les cinq processus existent: les échanges, le partage d'expériences, l'analyse, le débat et la création de nouvelles méthodes qui conduisent l'enseignant ou le futur enseignant à la formation professionnelle (Daele \& Charlier, 2006).

Les membres ont rejoint les communautés afin de:

- S'informer

- Communiquer/échanger des opinions avec leurs collèges

- S'informer et communiquer.

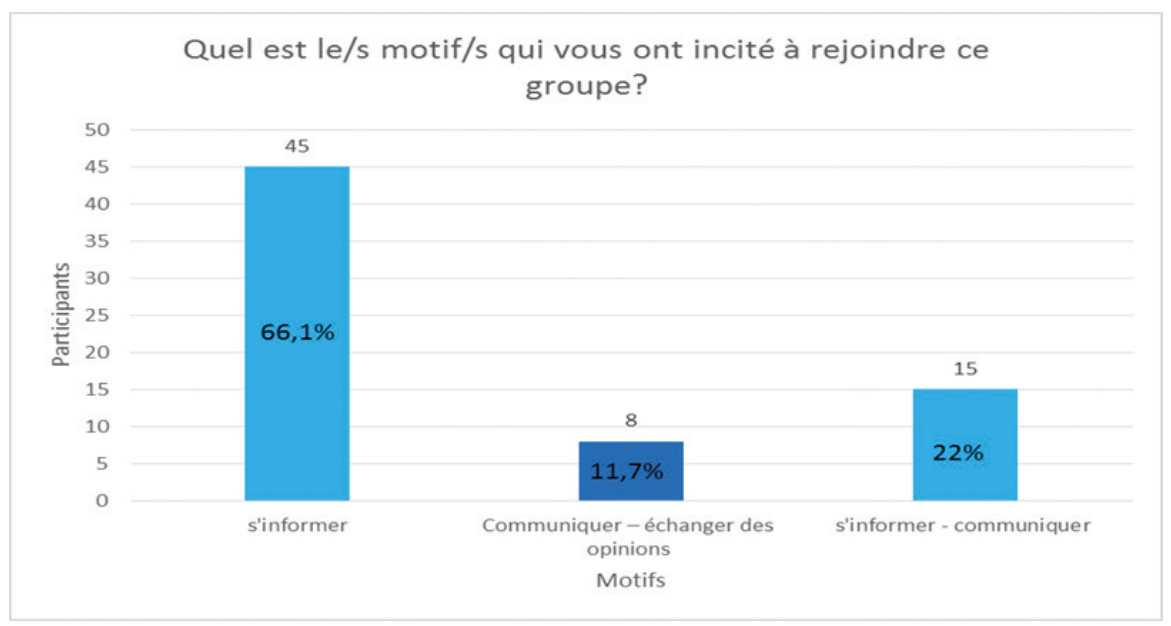

Figure 1

Les membres des communautés ont affirmé qu'ils élargissent leurs compétences communicationnelles qui sont très importantes pour leur formation professionnelle. Ensuite, nous avons remarqué qu'un nombre des membres de groupes préfèrent être passifs et non actifs. Même les membres qui ne participent pas aux débats virtuels, lisent les expériences 
et les questions posées des autres collègues, qui sont publiées et partagées à la communauté, et de cette façon, ils s'améliorent professionnellement parce que les pratiques des autres sont mises à leur disposition pour être adaptées, négociées ou réinvesties. Les membres passifs interagissent, quand ils utilisent les messageries privées. Nous constatons, alors, qu'ils échangent des points de vue, mais avec certains de leurs collègues. De plus, il y a une grande participation des membres aux discussions et l'existence de l'interaction est un élément important pour qu'un enseignant ou futur enseignant réussisse l'amélioration de ses compétences professionnelles.

Dès notre recherche nous avons conclu que les communautés de pratique aident les futurs enseignants et les enseignants à améliorer leurs compétences professionnelles. Elles leurs offrent:

- De nouvelles propositions concernant l'enseignement (nouvelles méthodes d'enseignement, séminaires).

- De l'échange des opinions.

- De la coopération parmi les membres.

- Des informations sur l'enseignement.

- De l'adaptation aux nouvelles pratiques d'enseignement/changement des pratiques utilisées.

- De l'acquisition d'expérience des collègues expérimentés.

- De nouvelles connaissances parmi les membres des groupes.

De notre recherche nous avons, aussi, constaté que les enseignants diplômés reviennent dans les communautés qui s'adressent exclusivement aux étudiants.

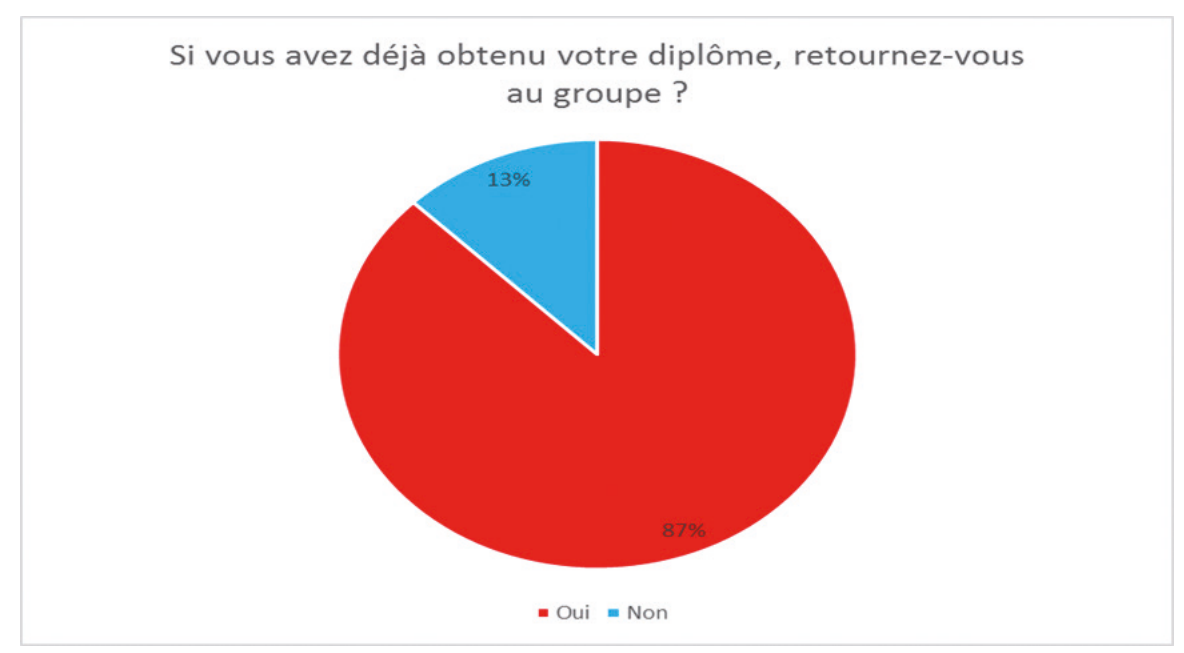

Figure 2 
Selon leurs réponses, ils reviennent dans les communautés parce que:

- Tous les groupes publient des annonces qui concernent les étudiants et les enseignants, aussi.

- Ils sont curieux.

- Ils échangent des idées/communiquent avec leurs collègues et les professeurs du département de la langue française et espagnole en Grèce, qui sont membres des groupes/aident les étudiants du département.

- Ils s'informent des annonces publiées sur les nouveautés concernant l'enseignement.

- Ils font de nouvelles connaissances et ils s'enrichissent personnellement.

- Ils s'informent sur les nouvelles méthodes/séminaires possibles/l'utilisation de nouveaux outils technologiques/virtuels concernant l'enseignement.

- Ils suivent les masters du département.

- Ils gardent le contact avec les nouveaux étudiants du département.

Nous concluons que la fréquence de participation aux groupes est indépendante de l'obtention du diplôme. Les étudiants et les enseignants s'intéressent aux sujets contemporains d'enseignement et aux changements possibles, et désirent se former professionnellement. Finalement, le retour aux communautés aide les enseignants expérimentés à garder le contact avec les nouvelles technologies et les derniers développements en didactique pour lesquels ils n'avaient pas eu l'occasion de s'informer, sans l'aide du réseautage social.

\section{Conclusion}

Cet article nous a permis de comprendre les raisons pour lesquelles les enseignants et les futurs enseignants de langue rejoignent les communautés de pratique sur Facebook. Nous avons réussi à identifier les bénéfices pour lesquels les enseignants et les étudiants de langues participent aux discussions à distance et de cette manière ils réfléchissent à leurs pratiques professionnelles. À l'aide de Facebook, les enseignants s'engagent dans des débats virtuels qui contribuent à l'amélioration de leurs compétences professionnelles. Le réseautage social assure la facilité de communication entre les membres à travers l'utilisation des outils interactifs. Nous avons réalisé que les communautés de pratique sur Facebook répondent aux besoins de leurs participants de se former professionnellement. La synthèse des échanges, les discussions à distance, les débats virtuels, la résolution des conflits sociocognitifs et finalement, l'interaction réalisée parmi les membres des communautés de pratique à travers le réseautage social élargissent les horizons cognitifs des enseignants qui demandent à parfaire leurs connaissances et leurs compétences. 
Plus concrètement, les communautés de pratique donnent la possibilité aux enseignants et aux étudiants de langue de:

- Valider/réinvestir leurs propres pratiques d'enseignement.

- Adopter de nouvelles pratiques d'enseignement.

De cette manière, cette recherche incite les enseignants et les futurs enseignants à fréquenter les groupes sur Facebook et les considérer comme des outils de développement professionnel. Cette recherche conduit les enseignants et les étudiants de langues à formuler leurs objectifs afin de participer à des communautés de pratique, à éveiller leur curiosité à fréquenter les groupes sur Facebook et à stimuler leur intérêt pour pouvoir progresser professionnellement.

\section{Références bibliographiques}

Blattner, G. \& M. Fiori. 2009. "Facebook in the Language Classroom: Promises and Possibilities" in Instructional Technology and Distance Learning (ITDL), vol. 6, $\mathrm{n}^{\circ}$ 1, 17-28: $<$ http://www.itdl.org/journal/jan_09/article02.htm>[21/01/2021].

Blattner, G. \& L. LomickA. 2012. "Facebook-ing and the Social Generation: A New Era of Language Learning. Médias sociaux et apprentissage des langues: (r)évolution?" in Apprentissage des langues et systèmes d'information et de communication (Alsic), vol. 15, $\mathrm{n}^{\circ} 1:<$ https://alsic.revues.org/2413>[15/01/2021].

Brown, J. S., Collins, A. \& P. Duguid. 1989. "Situated cognition and the culture of learning" in Educational Researcher, vol. 18, $\mathrm{n}^{\circ}$ 1, 32-42: <http://edr.sagepub.com/ content/18/1/32>[04/03/2021].

Butler, D., Lauscher, H., Jarvis-Selinger, S. \& B. Beckingham. 2004. "Collaboration and self-regulation in teachers' professional development" in Teaching and Teacher Education, vol. 20, 435-455.

Charlier, B. 2006. “Collaborer dans l'enseignement supérieur: apprentissages individuels et institutionnels" in AIPU, Université de Fribourg: <https://www.researchgate.net/ profile/Bernadette_Charlier/publication/228347585_Collaborer_dans_l'enseignement_suprieur_apprentissages_individuels_et_institutionnels/links $/ 09 \mathrm{e} \overline{4} 150 \overline{\mathrm{f}} 55 \mathrm{c} 1 \mathrm{ac} 202 \mathrm{a} 000 \overline{0} 00$. $\operatorname{pdf}>[\overline{11} / 04 / 2021]$.

Charlier, B. \& A. DAele. 2006. "Comprendre les communautés virtuelles d'enseignants" in Pratiques et recherches. Paris, L'Harmattan.

Charlier, B. \& D. Peraya (éds.). 2003. Technologie et innovation en pédagogie: dispositifs innovants de formation. Brussels, De Boeck Université.

CobB, P. \& E. YACKel. 1996. "Constructivist, emergent, and sociocultural perspectives in the context of development research" in Educational Psychologist, vol. 31, 175-190: <http:// www.tandfonline.com/doi/abs/10.1080/00461520.1996.9653265> [3/05/2021].

Collinson, V., Kozina, E., Kate Lin, Y., Ling, L., Matheson, I., Newcombe, L., et al. 
Anales de Filología Francesa, n. ${ }^{\circ}$ 29, 2021

FACEBOOK COMME PLATE-FORME DE DÉVELOPPEMENT DES COMPÉTENCES PROFESSIONNELLES DES...

2009. "Professional development for teachers: a world of change" in European Journal of Teacher Education, vol. 32(1), 3-19.

Daele, A. 2004. Développement professionnel des enseignants dans un contexte de participation à une communauté virtuelle: une étude exploratoire. Mémoire de DEA en Sciences de l'Éducation. Université catholique de Louvain.

Daele, A. 2010. "Conditions et vécu du conflit sociocognitif au sein d'une communauté virtuelle d'enseignants: proposition d'un cadre d'analyse et étude de cas" in Education \& Formation, vol. e-293, 65-80: <http://grms.uqam.ca/upload/files/Travail_en_communauts collaboration_et_partenariats_pour_le.pdf\#page $=65>[18 / 04 / 2021]$.

DAele, A. 2013. Discuter et débattre pour se développer professionnellement. Analyse compréhensive de l'émergence et de la résolution de conflits sociocognitifs au sein d'une communauté d'enseignants du primaire. Thèse de doctorat en Sciences de l'Education). Université de Genève, Genève:<http://archive-ouverte.unige.ch/unige:27065>[15/05/2021].

DAele, A. 2014. "Débattre en ligne pour se développer professionnellement. Adjectif, Analyses" in Recherches sur les TICE. Suisse: Université de Lausanne: $<$ http://www.adjectif.net/ spip/spip.php?article273>[22/05/2021].

DAY, C. 1999. Developing Teachers: The Challenges of Lifelong Learning. London, The Falmer Press.

Deaudelin, C., Dussault, M. \& M. Brodeur. 2003. "Human-Computer Interaction: A Review of the Research on its Affective and Social Aspects" in CJLT RCAT:<https://ejournals. library.ualberta.ca/index.php/cjlt/article/view/26556/19738>[23/05/2021].

Depover, C. \& L. Marchand. 2002. E-learning et formation des adultes en contexte professionnel. Belgique, De Boeck: <https://books.google.fr/books?hl=el\&lr=\&id=TCHNB$8 \mathrm{yOQwC} \& \mathrm{oi}=$ fnd \&pg=PA1\&dq=Depover $+\% 26+$ Marchand $+2002 \&$ ots $=2043 \mathrm{rMexvG} \&-$ sig=lpD3DxOmGpAT1C9e964Zit7d0_g\#v=onepage \&q=Depover\%20\%26\%20Marchand $\% 202002 \& f=$ false $>[22 / 05 / 2021]$.

Dillenbourg, P., Poirier, C. \& L. Carles. 2003. "Communautés virtuelles d'apprentissage: e-jargon ou nouveau paradigme?” in Taurisson, A. \& Sentini, A. (dir.). Pédagogies.net. Montréal, Presses Universitaires du Québec, 1-26.

Donnay, J. \& E. Charlier. 2006. Apprendre par l'analyse des pratiques. Initiation au compagnonnage réflexif. Namur, PUN-Editions du CRP.

Godwin-Jones, R. 2008. "Emerging Technologies: web-writing 2.0: Enabling, documenting, and assessing writing online" in Language Learning \& Technology, vol. 12, $\mathrm{n}^{\circ}$ 2, 7-13: $<$ http://llt.msu.edu/vol12num2/emerging.pdf $>$ [22/05/2021].

Greene, J. A. \& R. Azevedo. 2007. “A Theoretical Review of Winne and Hadwin's Model of Self-Regulated Learning: New Perspectives and Directions in Review of Educational Research, vol. 77(3), 334-372.

Gunawardena, C., Hermans, M., Sánchez, D., Richmond, C., Bohley, M. \& R. TuttLE. 2008. "A theoretical framework for building online communities of practice with social networking tools", U.S.A. University of New Mexico: <http://citeseerx.ist.psu.edu/viewdoc/ download?doi $=10.1 .1 .476 .6645 \&$ rep $=$ rep1\&type $=$ pdf $>[17 / 05 / 2021]$. 
Henri, F. \& B. Pudelko. 2006. "Le concept de communauté virtuelle dans une perspective d'apprentissage social" in Daele, A. \& Charlier, B. Comprendre les communautés virtuelles d'enseignants: Pratiques et recherches. Paris: L'Harmattan. 105-126.

Hou, H., Sung, Y. \& K. Chang. 2009. "Exploring the behavioral patterns of an online knowledge-sharing discussion activity among teachers with problem-solving strategy" in Teaching and Teacher Education, vol. 25(1), 101-108.

KARPATI, A. 2009. "Web 2 technologies for net native language learners: a "social CALL"' in $\operatorname{ReCALL}$, vol. 21, $\mathrm{n}^{\circ} 2,139-156$.

Karsenti, T., Depover, C. \& V. Komis. 2007. "Enseigner avec des technologies. Favoriser les apprentissages, développer des compétences" in Québec: Presses de l'université du Québec.

Mazer, J. P., Murphy, R. E. \& C. J. Simonds. 2007. 'I'll see you on 'Facebook': The effect of computer-mediated teacher self-disclosure on student motivation, affective learning and classroom climate" in Communication Education, vol. 56, $\mathrm{n}^{\circ} 1,1-17:<$ http://www.tandfonline.com/doi/abs/10.1080/03634520601009710\#.VzNKwYSLS1s>[19/05/2021].

MiLls, N. 2011. "Situated learning through social networking communities: The development of joint enterprise, mutual engagement, and a shared repertoire" in CALICO, vol. 28, $\mathrm{n}^{\circ} 2,345-368$.

MousA, A. 2018. "La construction des communautés virtuelles via Facebook et son potentiel en classes de langues: Enjeux et Perspectives" in Jordan Journal of Modern Languages and Literature, vol. 10, $\mathrm{n}^{\circ}$ 1, 63-76. <https://scholar.googleusercontent.com/scholar? $\mathrm{q}=-$ cache:zGUzGWS1zAAJ:scholar.google.com/+competences + professionnelles + des + enseignants + de + langues + etrangeres + communaut $\% \mathrm{C} 3 \% \mathrm{~A} 9+\mathrm{de}+$ pratique + Facebook\&hl $=$ el\&as sdt $=0,5>[24 / 05 / 2021]$.

Schön, D. 1983. “The reflective practicionner” in New York: Jossey Bass.

Sylla, N. \& L. De Vos. 2010. “Développement professionnel des enseignants au sein d'une communauté virtuelle" in Education \& Formation. Université de Bruxelles. < http://comsante.uqam.ca/upload/files/Travail_en_communauts_collaboration_et_partenariats_pour_ le.pdf\#page $=81>[24 / 05 / 2021]$.

Wenger, E. 2005. La théorie des communautés de pratique: Apprentissage, sens et identité. Presses de l'université de Laval.

YoRks, L. 2005. “Adult learning and the generation of new knowledge and meaning: creating liberating spaces for fostering adult learning through practitioner-based collaborative action inquiry" in Teachers College Record, vol. 107(6), 1217-12. 
FACEBOOK COMME PLATE-FORME DE DÉVELOPPEMENT DES COMPÉTENCES PROFESSIONNELLES DES...

\section{Annexes}

Questionnaire: Formation professionnelle sur Facebook

\section{Nom d'utilisateur Facebook}

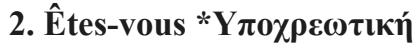

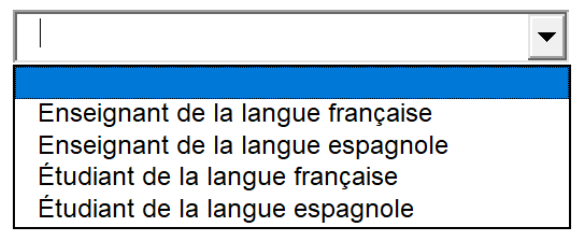

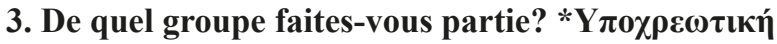

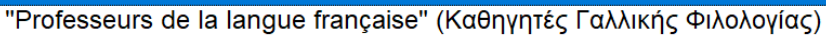

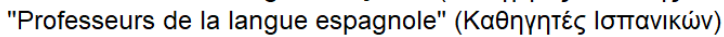

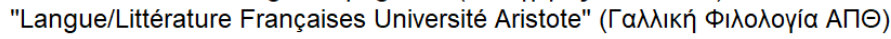

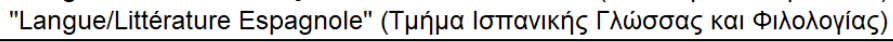

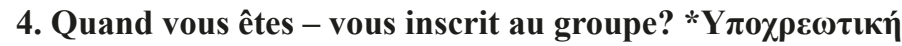

- $\quad$ Avant mon entrée à l'Université

- $\quad$ Dès mon entrée à l'Université

- $\quad$ Pendant mes études universitaires

- $\quad$ Après la fin de mes études universitaires

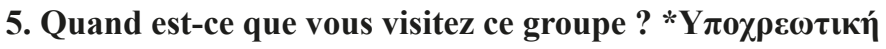

- $\mathrm{C}$ Tous les jours

- $\mathrm{C}$ Presque tous les jours

- $\mathbb{C}$ Une ou deux fois par semaine

- $\quad$ Une ou deux fois toutes les deux semaines

- $\mathbb{E}$ Une ou deux fois par mois

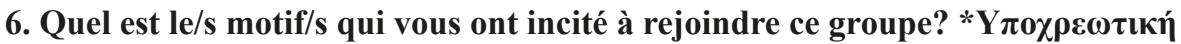

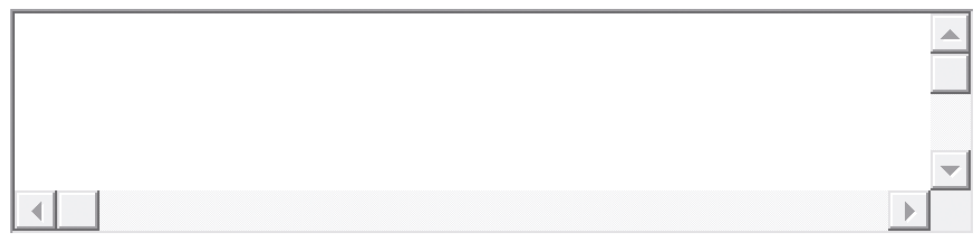




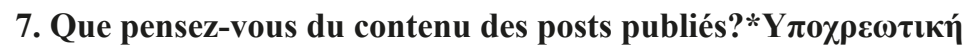

- $\mathrm{C}$ Ils sont très utiles

- $\mathrm{C}$ Ils sont peu utiles

- $\quad$ Ils ne sont pas utiles

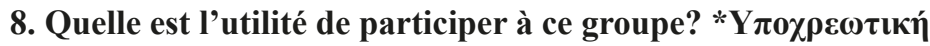

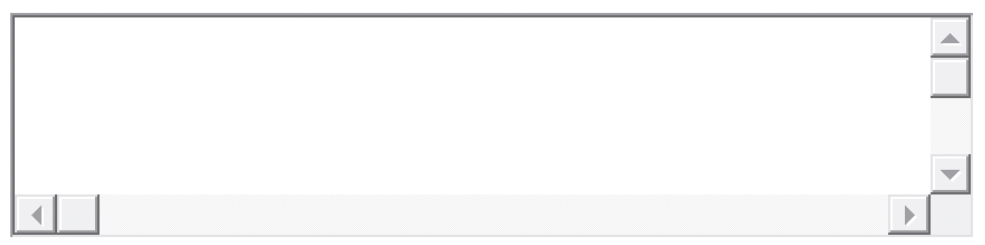

9. Si vous participez aux discussions, quel intérêt trouvez-vous? Qu'est-ce que

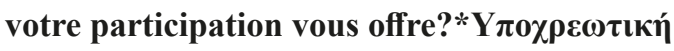

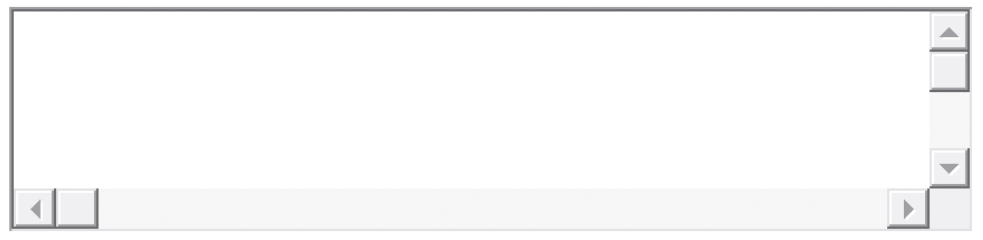

10. Pensez-vous que les collègues expérimentés aident/répondent aux novices?

- E Oui

- $\mathrm{C}$ Non

- $\mathrm{C}$ Je ne sais pas

11. D'habitude, quelles sont les questions que vous pouvez poser à ce groupe?

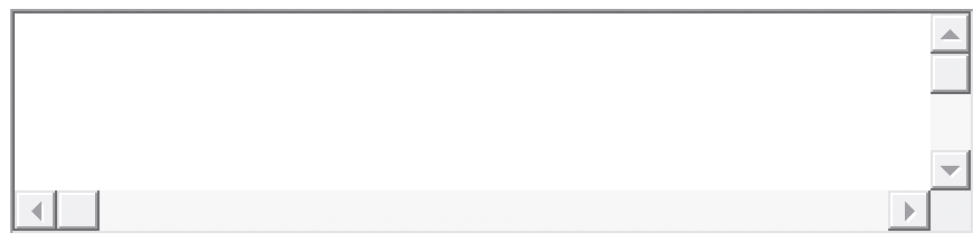

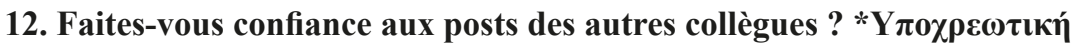

- $\mathrm{C}$ Oui

- $\mathrm{E}$ Non

- $\mathrm{E}$ Pas toujours 
FACEBOOK COMME PLATE-FORME DE DÉVELOPPEMENT DES COMPÉTENCES PROFESSIONNELLES DES...

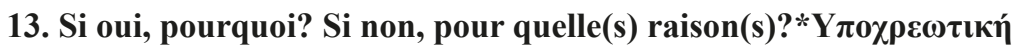

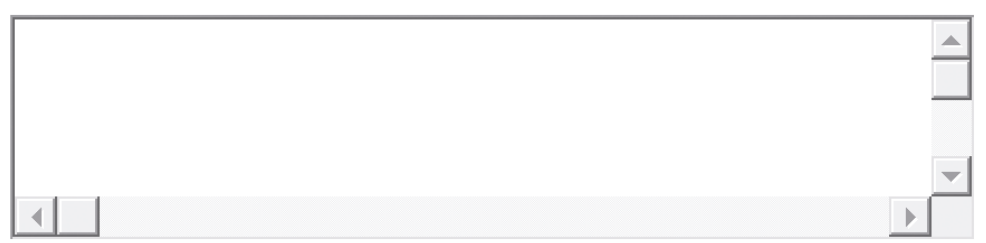

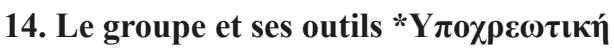

\begin{tabular}{l} 
"Tagger" un post \\
\hline Écrire un post \\
\hline Lire un post \\
Répondre aux commentaires d'un collègue \\
"Aimer" un post
\end{tabular}

15. Vos compétences professionnelles s'améliorent-elles suite à la fréquentation

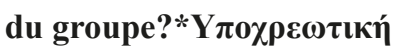

- $\mathrm{C}$ Oui, beaucoup

- $\quad$ Oui, assez

- $\mathrm{C}$ Pas trop

- $\mathrm{C}$ Non

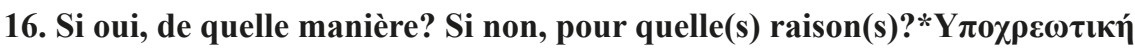

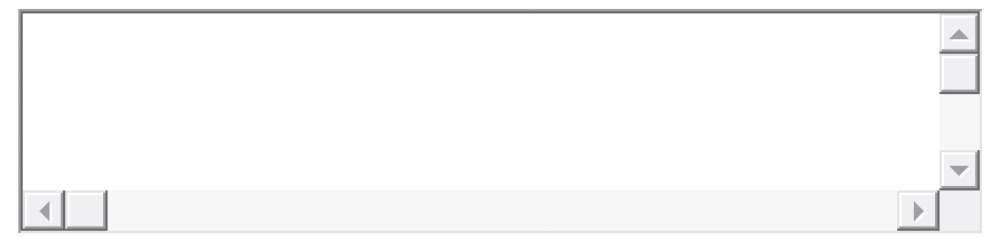

17. Si vous êtes professeur, vos pratiques d'enseignement se sont-elles améliorées tout au long de la fréquentation du groupe ?

- $\mathrm{C}$ Oui

- $\mathrm{C}$ Non 
18. Si oui, pourquoi? Si non, pour quelle(s) raison(s)?

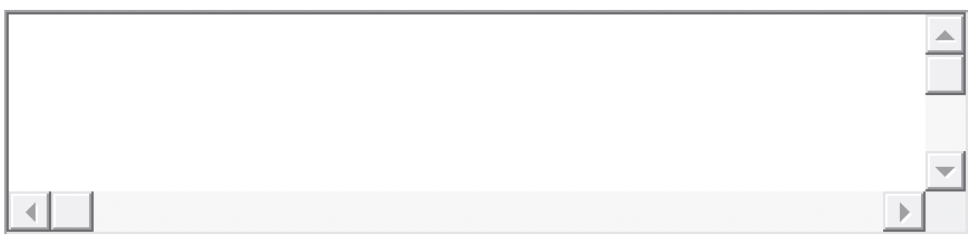

19. Si vous avez déjà obtenu votre diplôme, retournez-vous au groupe ?

- C Oui

- E Non

20. Si oui, pour quelle(s) raison(s)?

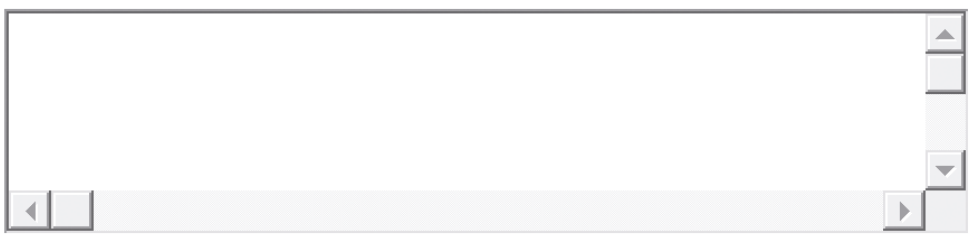

21. Quels sont les inconvénients que vous trouvez dans le groupe fréquenté

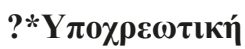

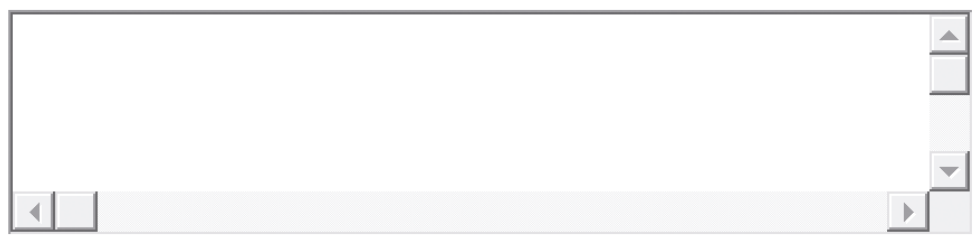


